\title{
Clinical Assessment and Thickness Changes of the Oblique and Multifidus Muscles Using a Novel Screening Tool and Exercise Program: A Randomized Controlled Trial
}

\author{
Brian Catania, Travis Ross, Bradley Sandella, Bradley Bley, and Andrea DiTrani Lobacz
}

\begin{abstract}
Context: Training and assessment of the abdominal and trunk muscles are widely used in the clinical setting. However, it is unknown what types of exercises are most effective in activation of both the global and local stabilizers in these regions. Objective: The purpose of this study was to establish the reliability of a novel clinical screening tool (sling screen) to assess the muscles of the abdomen and trunk. The second aim was to use the clinical screening tool and musculoskeletal ultrasound to compare the effects of a rotary-based exercise program that targets both the global and local muscles to the effects of a traditional exercise program on the activation of the abdominal and trunk muscles. Design: Double-blind, randomized controlled trial. Setting: Sports medicine facility. Participants and Interventions: Thirty-one healthy participants were randomly allocated to receive a single-session rotary-based or traditional "core" exercise program. Main Outcome Measures: The participants were assessed at the baseline and immediately postintervention. The primary outcome measures were muscle thickness examined by musculoskeletal ultrasound and clinical examination of muscle activation using a screening tool. The data were collected by blind assessors. Reliability and validity of a clinical screening tool (sling screen) were also assessed. Results: The analysis of the covariance tests showed a significant increase in oblique thickness for the rotary exercise group. All participants displayed a significant increase in multifidus thickness. The Wilcoxon signed-rank tests revealed a significant increase in clinical assessment scores in the rotary exercise group but not the traditional exercise group. Reliability of the sling screen ranged from moderate to good. Conclusion: This clinical trial provides evidence that a rotary-based exercise program may be more effective in producing increases in oblique muscle thickness than traditional "core" exercises in young, healthy adults. The sling screen tool was able to identify these muscle thickness changes. Future studies should investigate how these results correlate to injury risk, other populations, and also how to implement the sling screen into clinical practice.
\end{abstract}

Keywords: abdominal muscles, transversus abdominis, core slings, core muscles

Over recent decades, assessment and training of the trunk and abdominal muscles have gained tremendous popularity in the clinical setting. ${ }^{1-4}$ However, empirical data supporting the validity and effectiveness of these strategies have remained inconclusive. ${ }^{5-8}$ Endurance, strength, and asymmetry of the trunk muscles have been identified as modifiable risk factors that may help physically active patients rehabilitate and reduce the risk of musculoskeletal injuries. ${ }^{4,9,10}$ Asymmetries in the lumbar multifidus (MF) muscles and delayed recruitment and atrophy of the transverse abdominis (TA) have been identified in cases of low back pain and hip, groin, and thigh injuries. ${ }^{10-20}$ More recent studies have also demonstrated that injury-reduction programs focused on strengthening of these muscles are associated with lower incidence rates and improved functional measures. ${ }^{21,22}$ Hence, there exists an interest in establishing a means to effectively measure and train both the deep and superficial trunk and abdominal muscles. ${ }^{23}$

Controversy exists on how to clinically examine the trunk and abdominal muscles. Significant attention has been placed on the differentiation between the local and global muscle systems. ${ }^{24}$ Studies have shown that a "motor control" (local) exercise program

Catania and Ross are with the ChristianaCare, Newark, DE, USA. Sandella is with the ChristianaCare, Wilmington, DE, USA. Bley is with the Delaware Orthopaedic Specialists, Wilmington, DE, USA. DiTrani Lobacz is with the Department of Athletic Training, Neumann University, Aston, PA, USA. Catania (bcatania@ christianacare.org) is corresponding author. produces improved function and decreased pain in low back pain patients, ${ }^{25,26}$ while some have demonstrated a general (global) exercise approach to be superior in reducing disability. ${ }^{27,28}$ This local versus global model may have limited previous work in its applicability, and such a separation of muscle systems could be detrimental to continued growth in this area of research. ${ }^{24}$ It is more likely that both the local and global systems are important and contributing factors to function. ${ }^{29}$ Current assessment methods typically include global muscle endurance tests, such as the back extensor test, side plank test, and flexor endurance test,,${ }^{9,10}$ and musculoskeletal ultrasound (MSUS) has been used to examine the recruitment of the TA and MF muscles, known as motor control tests. ${ }^{30-32}$ While both methods of testing are commonly utilized due to practicality, ${ }^{9}$ the reliability and validity of most "core" muscle tests have not been established, which limits clinical usefulness. ${ }^{33}$ To our knowledge, no current validated screening tool has attempted to incorporate both the global and local trunk stabilizers.

A clinical tool with adequate reliability and validity that can be easily implemented is needed. ${ }^{33}$ To meet this clinical necessity, the concept of the "core muscle slings" may be an approach to satisfactorily examine this region. The slings were first presented by Vleeming, ${ }^{34,35}$ and they serve as anatomical keystone connections between the global muscles that provide stability and transfer of forces across multiple joints and the local stability provided by the TA and segmental MF. ${ }^{31,32,36}$ The slings are composed of the lateral sling (internal oblique [IO] and ipsilateral gluteus medius), 
posterior oblique sling (latissimus dorsi and contralateral gluteus maximus), and anterior oblique sling (external oblique [EO] and contralateral adductors), which together create a "core cylinder."37 Anecdotally, dysfunction in the global muscles leads to inhibition of the local stabilizers, and the slings are often described to influence the quality of motion via their myofascial connections. $^{36,38}$ This information may help the clinician identify related areas for targeted interventions. ${ }^{36}$ While cadaveric studies have identified other links between the muscles of the lowerextremity (hamstrings, adductors, and gluteus maximus) and the abdomen/trunk (erector spinae, latissimus dorsi, and rectus abdominis), ${ }^{38,39}$ validity and the functional assessment of the slings have yet to be examined in healthy participants. The use of MSUS may be a means of validating a tool that can more accurately assess trunk and abdominal muscle function, which has been previously cited as an acceptable method of measuring muscle activation. ${ }^{30,33,40,41}$

Contested theories of how to test the abdominal and trunk muscles lend to different methods on specific training.9,10 Training has been categorized into the same 2 aforementioned groups: global muscle exercises, including planks and endurance exercises, and motor control exercises, which involve volitional contractions of the MF and TA muscles. ${ }^{9,10,42,43}$ Both types of training have been shown to be useful in the rehabilitation process and injury prevention strategies. ${ }^{9,10,42-44}$ However, it remains to be determined if a rotation-based exercise program founded on the principles of the slings, which incorporates both global and local muscles, would be more effective than traditional exercises in activating the trunk and abdominal muscles.

Therefore, the first purpose of this study was to establish the reliability of a clinical screening tool in healthy participants. The second aim was to use the clinical screening tool and MSUS to compare the effects of a rotary-based exercise program that targets both the global and local muscles to a traditional exercise program on the activation of the abdominal and trunk muscles. Finally, we aimed to examine the validity of the clinical screening tool via MSUS.

\section{Methods}

\section{Study Design}

This study was a double-blind, randomized controlled trial. All participants provided written informed consent prior to group assignments and assessment, and the rights of all participants were protected. The ChristianaCare institutional review board approved the experimental procedures for this study. As a randomized controlled trial, the established recommendations provided by the Consolidated Standards of Reporting Trials statement were followed. All testing procedures were completed in physician examination rooms within a sports medicine facility.

\section{Participants}

Potential participants who had provided informed consent were considered for group assignment if they met the study inclusion criteria. The recruited participants included college students aged 18-26 years. The mean (SD) age, height, and weight of the participants was 20.8 (1.7) years, $169.7(8.9) \mathrm{cm}$, and 70.0 (12.7) kg, respectively. The exclusion criteria were current lumbar-region pain, surgery on the lower-extremity or spine in the past 6 months, or a history of neurological conditions (nerve injury, decreased sensation, and herniated disk with nerve involvement).

\section{Procedures}

Sling Screen. All participants underwent a clinical assessment (sling screen) to examine muscle activation. The sling screen is divided into 3 components: anterior, lateral, and posterior. The lateral sling was tested with hip abduction in a side-lying position with resistance to lateral knee and palpation along the fibers of the IO. A dowel rod was held by the subject, which was used to maintain the upper-extremity and trunk in a neutral position. The anterior oblique sling was tested while holding a dowel rod in the side-lying position, with the top leg resting on a foam roller to allow the bottom leg to adduct. Resistance was applied to the medial knee with palpation of the EO over the rib cage. The posterior oblique sling was tested in prone with the hip extended $10^{\circ}$, with resistance applied on the posterior heel, and palpation of contralateral latissimus dorsi. Each sling was graded on a 3-point Likert scale that considered compensations and quality of trunk and abdominal stabilization.

Muscle Thickness. A GE Logiq e (General Electric Medical Systems, Milwaukee, WI) MSUS device was used to obtain muscle thickness measures of the EO, IO, TA, and MF (Figure 1). ${ }^{30}$ Two sports medicine physicians, who were blinded to group allocation, performed the MSUS measurements. Upon presentation of a welldefined picture on screen, the image was saved, and measurements were obtained on the device with on-screen calipers. ${ }^{45}$ Ultrasound

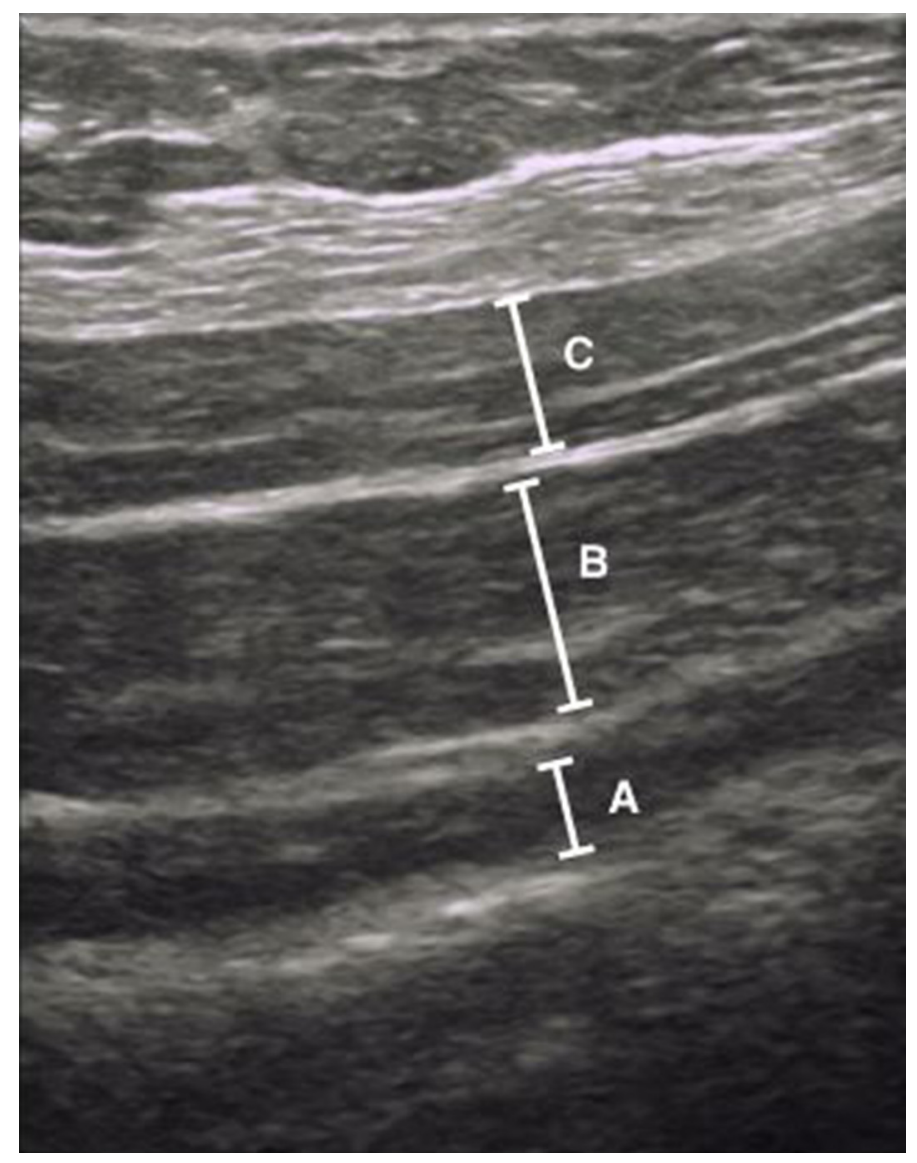

Figure 1 - Musculoskeletal ultrasound image of the trunk showing the (A) transverse abdominis, (B) internal oblique, and (C) external oblique muscles. 
imaging has been previously shown to be reliable for measurement of the TA and MF between clinicians and in multiple positions. ${ }^{46}$

Thickness measurements for the EO, IO, and TA were performed in a side-lying position. The location of the MSUS measures was 2-cm anterior and superior to the iliac crest on the particpant's dominant side. ${ }^{31}$ A $12-5 \mathrm{~Hz}$ linear probe was used in this procedure. In this position, the participants were asked to perform a series of 3 tasks for MSUS measurement of the EO, IO, and TA: inhalation, hip abduction, and hip adduction. Measurement of the MF was performed in the prone position with a pillow under the umbilicus to reduce excessive lordosis. ${ }^{33}$ The level of L3 was identified for horizontal MF measurement, and a 5-2 Hz curvilinear probe was used. The participants were asked to perform a contralateral hip extension movement during MSUS measurement of the MF.

Randomization. The participants were assigned to groups using an automated random number generator by an independent researcher who was not involved with the data collection process. Randomization occurred following the baseline data collection. Randomization of the participants occurred into 2 groups, as follows: a control group that received a traditional abdominal exercise program (CON) and an intervention group that performed a rotary-based exercise program (Sling Activation Session [SAS]).

Blinding. In this study, all assessors were blinded to the allocation of the participants within their respective groups. The participants were also blinded to their group assignment.

Sample Size Calculation. The sample size and power calculations were performed using $G^{*}$ Power (version 3.1.7; Dusseldorf, Germany). The calculations were based on previous research detecting a significant difference in the deep trunk muscles between, ${ }^{45}$ assuming a 2 -tailed test, an alpha level of .05 , and a desired power of $80 \%$. The estimated desired sample size was 14 participants per group.

Interventions. Following randomization, the participants were directed to a physical therapist for the exercise intervention. The participants assigned to the CON group completed traditional "core" exercises: sit-up, double-leg bridge, bicycle crunch, V-up, single-leg bridge with straight-leg raise, swimmer, side plank, and prone plank. The intervention for the experimental group (SAS) consisted of rotary-based exercises, including the side-lying thoracic rotation, lounge and pound, side-lying skater, upside down turtle, row dog, side high plank windmill, and kneeling chop/thrust (Figures 2-8). Ten repetitions were completed for all exercises, except for planks, which were completed as 2 sets of 15 seconds. Both programs took approximately 8 minutes for completion. Cuing was provided during performance to ensure that exercises were completed properly. Each exercise program duration lasted between 8 and 10 minutes, whether SAS or CON. Immediately following the single-session interventions, MSUS measurements and sling screen assessments were repeated.

Assessment of Sling Screen Reliability. Two physical therapists (B.C. and T.R.) with an average of 15 years of physical therapy practice participated in the reliability portion of this study. Thirtytwo healthy participants were evaluated during 1 testing day. Both physical therapists evaluated each participant using the sling screen in a randomized order. Each of the physical therapists and participants were blinded to the results of the other examiner, and testing was performed behind privacy curtains. The 2 examiners independently placed the participant into the testing positions,

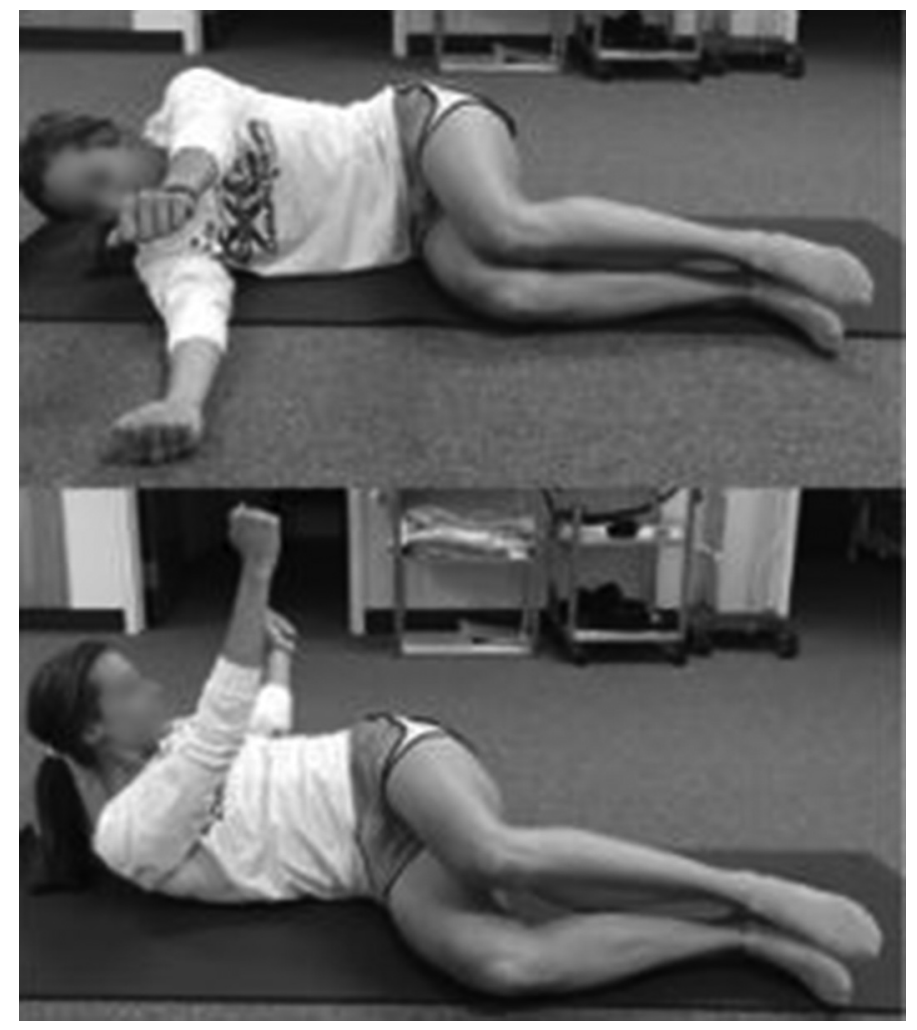

Figure 2 - Side-lying thoracic rotation.

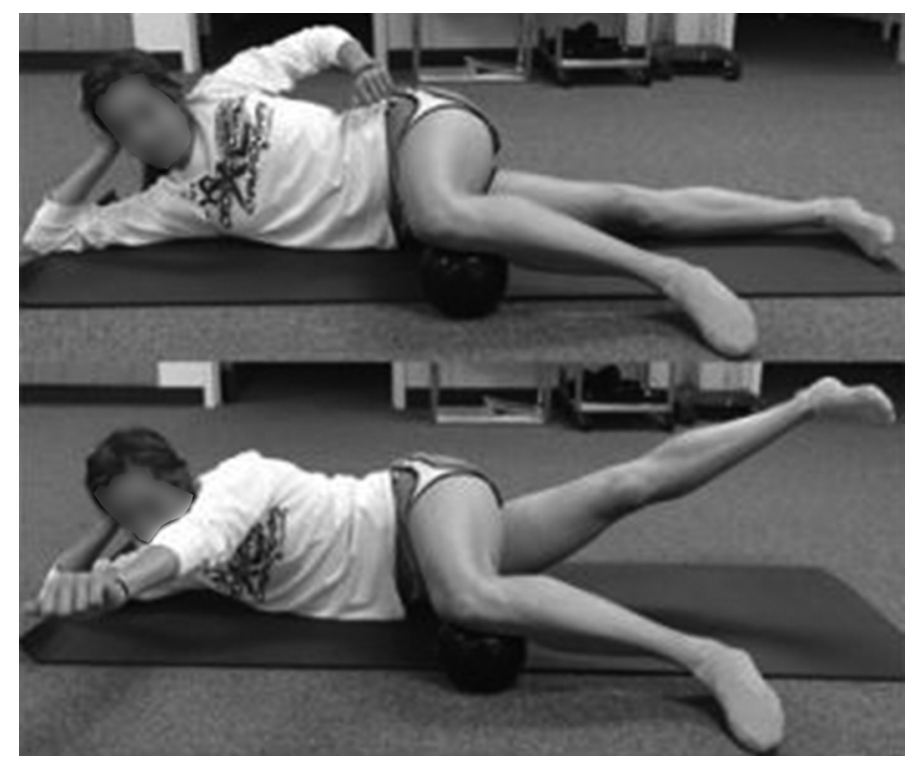

Figure 3 - Lounge and pound.

instructed the participant on the respective tasks, and recorded the scores on the testing sheet.

\section{Statistical Analysis}

All statistical analyses were performed using SPSS (version 22.0; IBM Corp, Armonk, NY). The significance levels were set at alpha $<.05$ for all analyses. For the reliability portion of the study, 


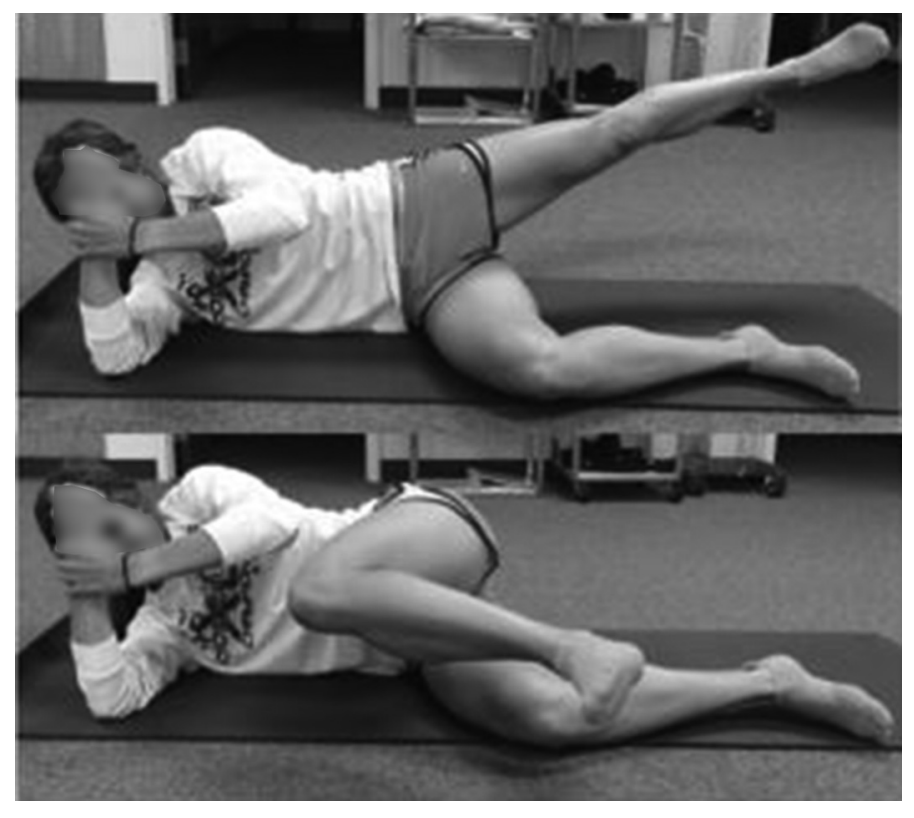

Figure 4 - Side-lying skater.

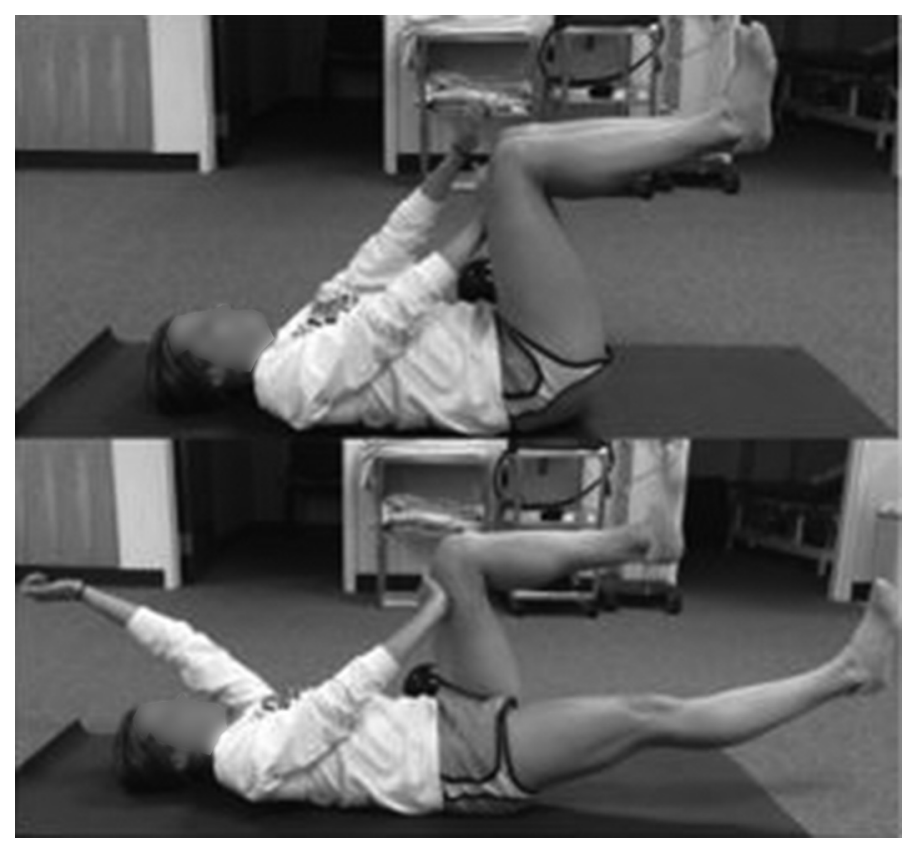

Figure 5 - Upside down turtle.

Kappa coefficients were calculated to examine agreement between the 2 physical therapists that completed the sling screen assessments and to account for chance effects in scoring. A 3-grade rating system was used for scoring performance. Typically, Kappa coefficients are interpreted according to the following distributions: excellent agreement (>.80), substantial agreement (.61-.80), moderate agreement (.41-.60), fair agreement (.21-.40), and slight agreement (.00-.20). ${ }^{47}$ For muscle thickness, analysis of covariance was used to adjust for group differences at the baseline, and the between-subjects factor was group (SAS and CON). Changes in sling screen scores were

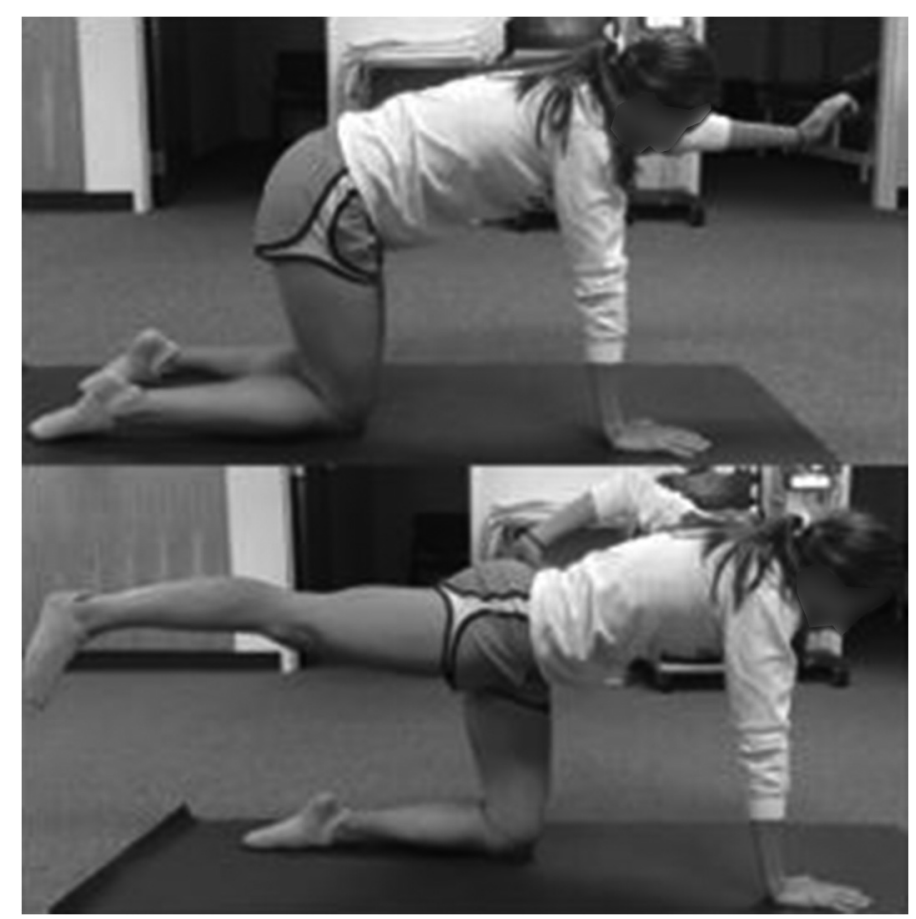

Figure 6 - Row dog.

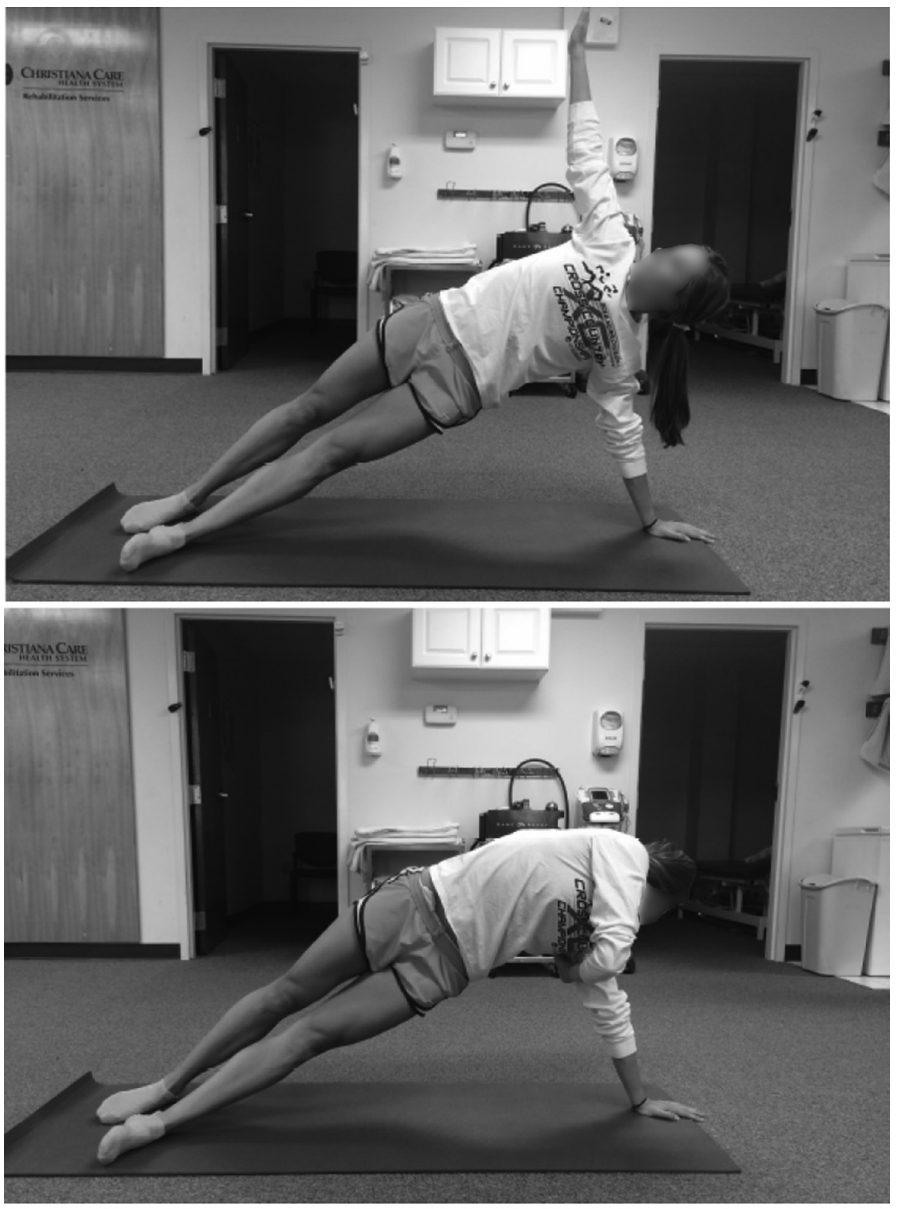

Side Plank Windmill

Figure 7 - Side Plank Windmill. 

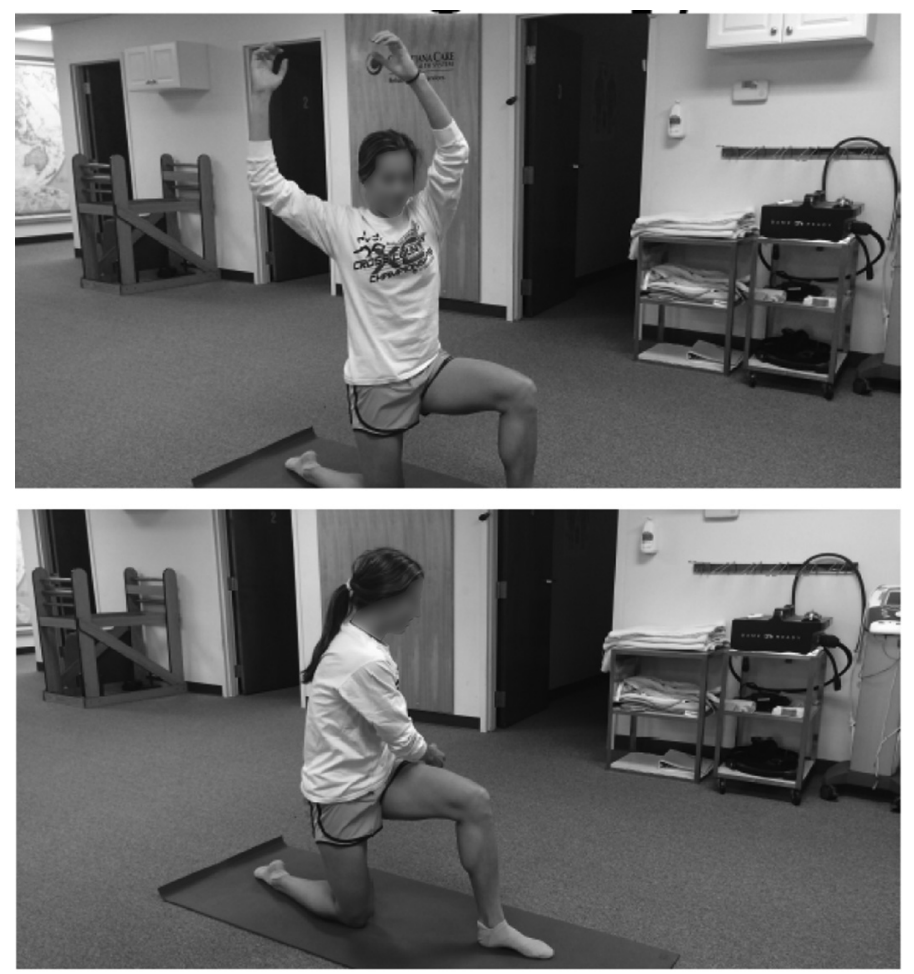

Kneeling chop/thrust

Figure 8 - Kneeling chop/thrust.

assessed using Wilcoxon signed-rank tests for each group (SAS and CON). The relationship between the sling screen scores and muscle thickness measures was assessed using a Spearman correlation. The effect sizes were calculated and interpreted as weak $(<0.2)$, small $(0.21-0.5)$, medium (0.51-0.8), or large $(>0.8)$.

\section{Results}

Recruitment commenced in October 2015 and was completed by August 2016. A total of 31 participants met the inclusion criteria and were able to be randomized into the data collection process (Figure 9). Fifteen participants were allocated to the CON group and 16 to the SAS group. The participant information is displayed in Table 1.

\section{Sling Screen Reliability}

Reliability of the sling screen was shown to range from "moderate" to "good." The Kappa values were calculated as .657 for the anterior sling, .497 for the posterior sling, and .762 for the lateral sling.

\section{Pre-Post Intervention Muscle Thickness}

A significant group difference was observed postintervention for both the IO $(F=19.885, P=.04)$ and EO $(F=20.684, P=.02)$ muscles during their respective testing movements, with the SAS group demonstrating a significantly greater increase in muscle thickness (Table 2). A medium effect size was found for betweengroup comparisons. A significant effect for condition, but not group, was observed for the MF, as all participants displayed an increase in thickness from the baseline (experimental group mean, 2.70 [0.41]; 95\% confidence interval [CI], 2.5-2.9; CON group mean, 2.44 [0.42]; 95\% CI, 2.2-2.7) to postintervention (experimental group mean, 2.85 [0.57]; 95\% CI, 2.6-3.1; CON group mean, 2.44 [0.42]; 95\% CI, 2.4-2.9) measurements $(F=4.57, P=.04)$. No significant differences were observed for the TA $(P>.05)$.

\section{Pre-Post Intervention Sling Screen Scores}

A statistically significant increase in sling screen scores was observed in the SAS group for the anterior $(z=-2.07, P=.04)$, posterior $(z=-2.53, P=.01)$, and lateral slings $(z=-2.01, P=.05)$, but no significant difference from preintervention to postintervention was demonstrated in the CON group for the anterior $(z=-1.82$, $P=.07)$, posterior $(z=-.333, P=.74)$, or lateral slings $(z=-.272$, $P=.79)$.

\section{Correlations Between Muscle Thickness and Sling Screen Scores}

The anterior sling screen scores were significantly correlated with thickness of the MF muscle during the right hip-extension maneuvers $(P=.003, r=.510)$. The anterior sling scores were also significantly correlated with the thickness of the EO during the testing maneuver $(P=.048, r=.358)$. No significant correlations existed between the posterior and lateral sling screens and muscle thickness.

\section{Discussion}

The results of this study indicate that the sling screen is a method of acceptable reliability. It is a quick and affordable means of oblique and MF muscle assessment that could easily be implemented in various settings. Through use of the sling screen, the examiners were able to identify muscle changes induced by the intervention exercises and scores correlated with MSUS thickness measurements. This provides initial support for the validity of the core slings. A strength of the sling screen is that it assesses the trunk as a functional unit, where muscle activation is associated with the motor task performed. ${ }^{29,48-50}$ The sling screen was designed to incorporate both the local and global systems, and we expected that all "core" muscles would contribute to stability during testing. This tool incorporates the synchronization of both muscle groups to support the trunk during movement and provides the clinician with an ability to identify potential deficits in either system. ${ }^{49}$ Future research should investigate how patient outcomes are related to the perception of stability and the sling screen.

\section{Muscle Thickness Changes}

We observed rotation exercises (SAS) to be superior in improving clinical scores of muscle activation and increasing MSUS thickness values in the intervention group. The SAS was designed to target the "sling" muscles, and this study was able to identify a number of significant differences between the SAS and CON groups following the exercise interventions. The thickness of the IO and EO muscles increased significantly more in those individuals who completed the SAS exercises. As rotators of the trunk, this demonstrates that exercise selection focused on dynamic rotational movements is superior in oblique muscle activation during completion of hip abduction and adduction tasks compared with traditional abdominal exercises. While other studies have failed to identify significant increases in the oblique muscles following motor control exercises and other interventions, ${ }^{51,52}$ this was the first study to 


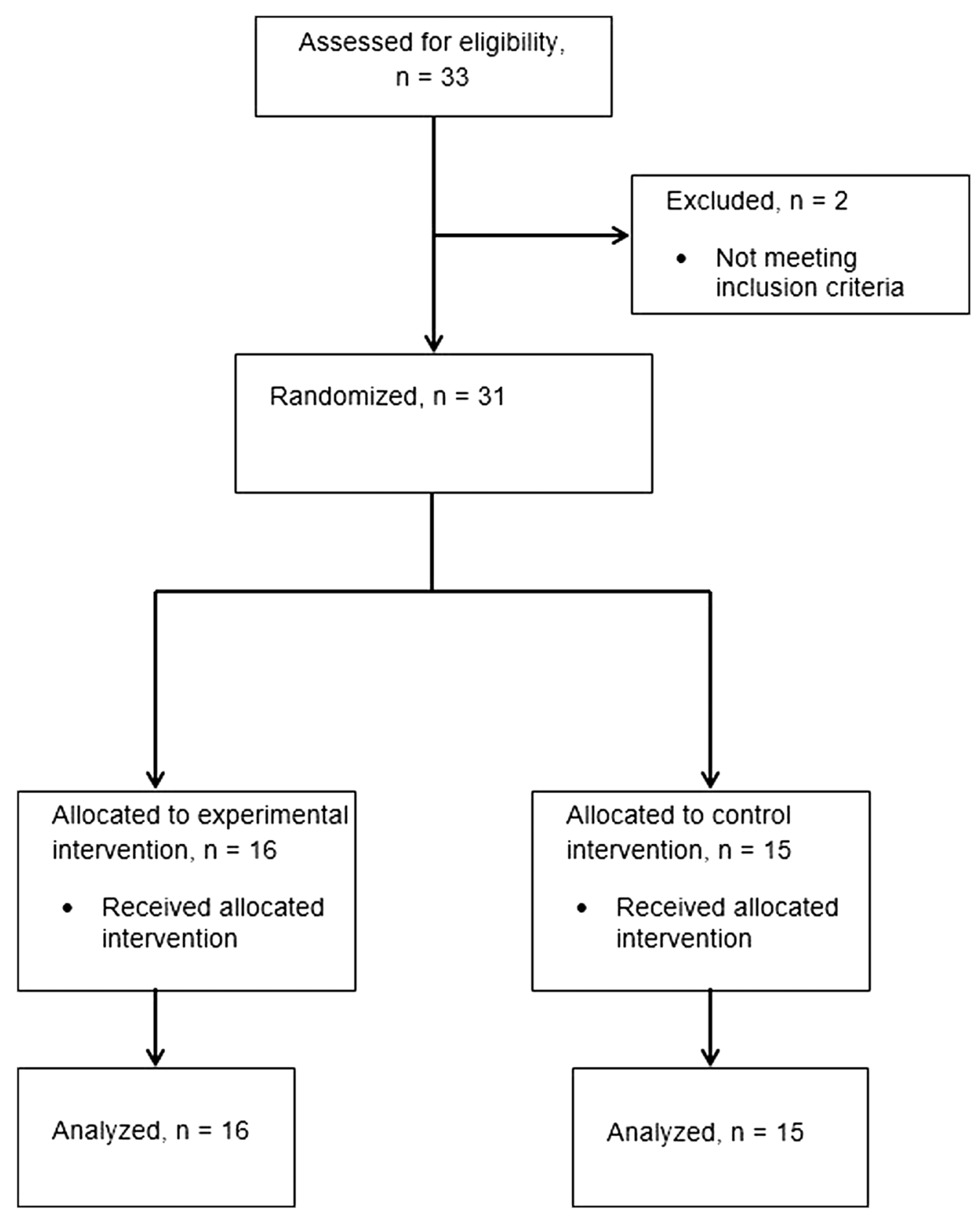

Figure 9 - Flow diagram of the study.

specifically evaluate rotation-based versus traditional abdominal exercises in a healthy population.

Similar to previous studies, we did not observe significant changes in the tranverse abdominis (TA). TA thickness has been demonstrated to correlate significantly with $\mathrm{EMG},{ }^{41}$ yet a large amount of variance has been displayed in both its recruitment and size. ${ }^{53-55}$ While the IO muscle likely undergoes significant stretch during rotary movements, due to its oblique orientation, the same may not occur to the TA owing to its transverse fiber alignment. ${ }^{56,57}$ In addition, the TA has demonstrated a contraction pattern closely resembling the IO during various exercises, which suggests that it may be active during the SAS exercises, ${ }^{58,59}$ but further investigation is needed based on the current results. Studies implicating the TA in pathology have most often been relative to timing delays in activation, rather than compromised muscle contraction. ${ }^{56,60-62}$
Also, we did not instruct the participants to perform abdominal bracing or hallowing techniques during testing, which have been used in previous studies to specifically highlight TA muscle activity. ${ }^{63-65}$ However, a dominant role of the TA may not exist in maintaining trunk stability, with the other global and local muscles serving as the main contributors to trunk stability. ${ }^{29,66,67}$

While we did not observe group differences in MF activation, both the SAS and CON participants demonstrated increased thickness. Testing demands may have been too small to identify group differences, as only small increases in MF activity are necessary to provide stability to the spine. ${ }^{68} \mathrm{We}$ expected an increase in MSUS MF thickness following the single-session SAS intervention, ${ }^{56,69-71}$ and a few explanations might exist for the increase observed in both groups. The MF may have been activated in all participants during the anterior oblique sling testing, as it occurs within the transverse 
Table 1 Participant Demographics

\begin{tabular}{lccc}
\hline Variable & All participants $(\mathbf{n}=\mathbf{3 1})$ & SAS group $(\mathbf{n}=\mathbf{1 6})$ & CON group $(\mathbf{n}=\mathbf{1 5})$ \\
\hline Sex, $\mathrm{n}(\%)$ & & & $5(33.3)$ \\
Male & $10(32.3)$ & $5(31.3)$ & $10(66.7)$ \\
Female & $21(67.7)$ & $11(68.8)$ & $20.7(1.8)$ \\
Age, $\mathrm{y}$ & $20.8(1.7)$ & $21.0(1.5)$ & $69.1(14.0)$ \\
Weight, $\mathrm{kg}$ & $70.0(12.7)$ & $71.2(11.2)$ & $1.69(0.89)$ \\
Height, $\mathrm{m}$ & $1.70(0.09)$ & $1.69(0.09)$ & \\
\hline
\end{tabular}

Abbreviations: SAS, sling activation session; CON, control. Note: Values are Mean (SD).

Table 2 Muscle Thickness Measured With Musculoskeletal Ultrasound

\begin{tabular}{llr}
\hline Group & CON $(\mathbf{n}=\mathbf{1 5})$ & SAS (n = 16) \\
\hline External Oblique $(\mathrm{CI}), \mathrm{cm}$ & $0.671(0.036),(0.6-0.7)$ & $0.820(0.035),(0.7-0.0)^{*}$ \\
Internal Oblique $(\mathrm{CI}), \mathrm{cm}$ & $1.038(0.308),(0.9-1.1)$ & $1.209(0.282)(1.1-1.3)^{*}$ \\
\hline
\end{tabular}

Abbreviations: SAS, sling activation session; CON, control; CI, confidence interval. Note: Values are Mean (SE).

*Significant difference between groups postintervention.

plane. Surrounding musculature should counter-rotate same side rotation, which would likely elicit MF contraction. Dorsally, the MF performs the same functions as the EO, so these muscles work synergistically from anterior to posterior to maintain stability during contralateral rotation and ipsilateral side bending.

The MF thickness has been increased following both a targeted exercise protocol and a general program, ${ }^{51}$ and a recent systematic review was also unable to determine which exercise was most effective in enhancing MF activity. Thus, increased thickness may occur when performing a series of exercises, especially those that include a progression from motor control to more functional movements. ${ }^{72}$ Much attention has been placed on the physical properties of the MF muscle, yet cross-sectional area has not been significantly associated with functional improvements measured via patient-reported outcomes in low back pain (LBP) patients, ${ }^{73}$ and similar reports have been documented for the TA. ${ }^{52}$ Thus, observable changes in morphology may not be required to assess progress in the clinical setting.

\section{Relationship Between Clinical and MSUS Measures}

In addition to thickness changes, we also identified a significant relationship between the anterior sling screen score and the L3 MF muscles, with higher scores related to increased thickness. Koppenhaver et $\mathrm{al}^{56}$ observed similar clinically related results following a longer term exercise program that established a relationship between MF thickness and improved disability in LBP patients, demonstrating the functional importance of this muscle. The results of our study are representative of the myotomal connection between the L3 nerve root and the adductor muscles. ${ }^{74,75}$ Knee extension is often the practiced L3 myotome test in the clinical setting, but our results support the assessment of the secondary muscles known to be anatomically supplied by these nerve roots. We measured MF thickness at the L3 level, whereas most studies that have identified increases in thickness have been reported at the L4-L5 and L5-S1 levels. ${ }^{76}$ Studies have also cited the reliability and validity of clinical tests, such as the MF lift test, to be specific to the level of the spine being tested. ${ }^{33}$ Continued research is needed to establish this relationship at other vertebral levels, and clinicians should also take this into consideration in the clinical setting and in future research.

A significant relationship was also observed between the EO and hip adductor test score, which may provide evidence for validation of the anterior oblique sling. These results add to the growing body of literature that suggests a connection between the proximal "core" and lower-extremity function. Focus on the myofascial chains in treatment and rehabilitation has become popular in the clinical setting, ${ }^{39}$ and our results support a connection between the EO and adductors. Although histologic studies suggest a mechanical function of fascia, ${ }^{77,78}$ much of the evidence regarding myofascial links has been anecdotal. ${ }^{39}$ However, recent studies have provided evidence for a number of the myofascial chains defined by Myers. ${ }^{38}$ Cadaveric evidence has supported the existence of links between the muscles of the lower-extremity (hamstrings, adductors, and gluteus maximus) and the abdomen/ trunk (erector spinae, latissimus dorsi, and rectus abdominis). ${ }^{76}$ Thus, our results may add to the growing body of literature that identifies such links, yet functional implications are yet to be determined via robust research studies.

While a significant relationship was not observed between the IO and sling screen scores during hip-abduction testing (lateral sling), it is possible that an increase in muscle thickness during performance may not be optimal. In the testing position, the IO remains in a neutral position and a contraction (shortening) of the muscle would result in an anterior pelvic tilt. Ideally, the IO should allow for neutral stabilization of the pelvis to permit optimal gluteus medius activation to abduct the hip. This would produce a higher lateral sling screen score during assessment of the hip abduction and may translate to a stronger gluteus medius contraction during testing and in the weight-bearing position, without necessarily altering the muscle thickness.

The impact of exercise type on activation of the oblique muscles has been documented, most often when comparing traditional sit-ups and crunches. ${ }^{58,79-81}$ To our knowledge, this was the first study to compare more dynamic, rotation-based exercises to a traditional exercise program and measure changes in clinical 
assessment scores and muscle thickness. Determining the influence of abdominal exercise selection on muscle activation is important to clinicians who incorporate training into interventions designed for injury prevention and rehabilitation, ${ }^{82}$ and it has been shown that all "core" exercises do not produce equal muscle activation. ${ }^{83}$ From a clinically relevant perspective, the sling screen scores from examiner assessments were significantly improved following the exercise interventions in the SAS group, but not the CON group. The clinical efficacy of "core" exercise programs is promising, ${ }^{22,84}$ and the sling screen may allow clinicians to appropriately identify patients that could benefit from such interventions. For instance, a 6-week intervention utilized as a warm-up in a previous study was able to positively influence lower-extremity muscle asymmetries and improve jumping performance, ${ }^{84}$ while other studies have identified improved knee flexor and extensor strength measures following core stability programs. ${ }^{85,86}$ More high-quality research in this area is needed, but preliminary evidence indicates that the "core" is a relevant component of lower-extremity function.

\section{Limitations}

While MSUS has been accepted as a reliable tool in the assessment of abdominal and trunk activation, ${ }^{87}$ fine-wire EMG remains the gold standard for evaluation of muscle contraction. ${ }^{88}$ Our results may indicate muscle activation changes, but some neuromuscular properties may be overlooked due to the instrumentation used in this study. However, MSUS remains a more realistic tool that can be used in the clinical setting, and this makes this study more applicable to health care practitioners. Clinicians are advised to proceed with caution, as comparison with the gold standard may produce different results. In addition, our recruitment of healthy, college-aged students may limit extrapolation to other age groups and patient populations, especially with regard to lack of significant group differences observed in the TA and MF muscles. In addition, physical fitness level was not evaluated in this study and could have impacted our results. While the SAS program appeared to activate the oblique muscles more than the CON group, practitioners should exercise caution when applying this program, as the exercises have not been used in other clinical trials and validity is lacking. Furthermore, while this study supports the existence of the core slings, a cadaveric study of the slings has not been previously reported, and proof of fascial connections remains to be established in the laboratory setting. Finally, the sling screen should also be subjected to further evaluation to examine its effectiveness in other patients and settings different from the population used in this study.

\section{Conclusion}

While clinical integration far exceeds empirical evidence supporting the importance of trunk and abdominal muscle assessment and relevance to function, our results add to the growing body of knowledge. This study shows that a rotary-based core exercise program may be more effective than a traditional core exercise program to recruit the oblique muscles, while also showing moderate to good reliability of the core sling screen tool. Muscle thickness was significantly increased for the oblique muscles in the rotarybased SAS group, demonstrating that exercise selection is critical in "core" muscle activation. The SAS is an exercise program that can be easily implemented as part of a rehabilitation, warm-up, or training protocol within various settings. The immediate effects of the SAS may be more beneficial compared with traditional attempts to activate the trunk and abdominal muscles, yet this remains to be determined. The sling screen was able to identify the participants who completed the SAS protocol, and demonstrated moderate to good reliability. It may be useful for clinicians to adopt these tools when aiming to improve muscle activation, but future study is warranted. Although trunk and abdominal muscle function has been thoroughly researched, a clinical tool with links to the lower-extremity that is both reliable and practical has been lacking. Future studies should continue to examine the usefulness and efficacy of the sling screen and the SAS exercise program, especially with respect to rehabilitation, injury risk reduction, and use with other patient populations.

\section{Acknowledgments}

The study protocol was proved by the institutional review board of Christiana Care Health System in Newark, Delaware, CCC No. 35034, DDD No. 602910. This study was registered at ClinicalTrials.gov (NCT3539211).

\section{References}

1. Bliss LS, Teeple P. Core stability: the centerpiece of any training program. Curr Sports Med Rep. 2005;4(3):179-183. PubMed ID: 15907272 doi:10.1097/01.CSMR.0000306203.26444.4e

2. Akuthota V, Ferreiro A, Moore T, Fredericson M. Core stability exercise principles. Curr Sports Med Rep. 2008;7(1):39-44. PubMed ID: 18296944 doi:10.1097/01.CSMR.0000308663.13278.69

3. Reiman MP. Trunk stabilization training: an evidence basis for the current state of affairs. J Back Musculoskelet Rehabil. 2009;22(3): 131-142. PubMed ID: 20023342 doi:10.3233/BMR-2009-0226

4. Huxel Bliven KC, Anderson BE. Core stability training for injury prevention. Sports Health. 2013;5(6):514-522. PubMed ID: 24427426 doi:10.1177/1941738113481200

5. Friedrich J, Brakke R, Akuthota V, Sullivan W. Reliability and practicality of the core score: four dynamic core stability tests performed in a physician office setting. Clin J Sport Med. 2017;27(4):409414. PubMed ID: 28653966 doi:10.1097/JSM.0000000000000366

6. Willardson JM. Core stability training: applications to sports conditioning programs. J Strength Cond Res. 2007;21(3):979. PubMed ID: 17685697

7. Cissik JM. The role of core training in athletic performance, injury prevention, and injury treatment. Strength Cond J. 2011;33(1):10-15. doi:10.1519/SSC.0b013e3182076ac3

8. Sherry M, Best T, Heiderscheit B. The core: where are we and where are we going? Clin J Sport Med. 2005;15(1):1-2. PubMed ID: 15654183 doi:10.1097/00042752-200501000-00001

9. Brumitt J, Matheson J, Meira EP. Core stabilization exercise prescription, part I: current concepts in assessment and intervention. Sports Health. 2013;5(6):504-509. PubMed ID: 24427424 doi:10. 1177/1941738113502451

10. Brumitt J, Matheson J, Meira EP. Core stabilization exercise prescription, part 2: a systematic review of motor control and general (global) exercise rehabilitation approaches for patients with low back pain. Sports Health. 2013;5(6):510-513. PubMed ID: 24427425 doi:10.1177/1941738113502634

11. Gildea JE, Hides JA, Hodges PW. Size and symmetry of trunk muscles in ballet dancers with and without low back pain. J Orthop Sports Phys Ther. 2013;43(8):525-533. PubMed ID: 23633627 doi:10.2519/jospt.2013.4523

12. Hides JA, Brown CT, Penfold L, Stanton WR. Screening the lumbopelvic muscles for a relationship to injury of the quadriceps, hamstrings, and adductor muscles among elite Australian football 
league players. J Orthop Sports Phys Ther. 2011;41(10):767-775. PubMed ID: 21891873 doi:10.2519/jospt.2011.3755

13. Hides J, Hughes B, Stanton W. Magnetic resonance imaging assessment of regional abdominal muscle function in elite AFL players with and without low back pain. Man Ther. 2011;16(3):279-284. PubMed ID: 21185218 doi:10.1016/j.math.2010.11.009

14. Cowan SM, Schache AG, Brukner P, et al. Delayed onset of transversus abdominus in long-standing groin pain. Med Sci Sports Exerc. 2004;36(12):2040-2045. PubMed ID: 15570137 doi:10.1249/ 01.MSS.0000147587.81762.44

15. Hodges PW, Richardson CA. Delayed postural contraction of transversus abdominis in low back pain associated with movement of the lower limb. Clin Spine Surg. 1998;11(1):46-56.

16. Djordjevic O, Djordjevic A, Konstantinovic L. Interrater and intrarater reliability of transverse abdominal and lumbar multifidus muscle thickness in subjects with and without low back pain. J Orthop Sports Phys Ther. 2014;44(12):979-988. PubMed ID: 25366083 doi:10. 2519/jospt.2014.5141

17. Haddas R, Sawyer SF, Sizer Jr PS, Brooks T, Chyu M, James CR. Effects of volitional spine stabilization and lower extremity fatigue on trunk control during landing in individuals with recurrent low back pain. J Orthop Sports Phys Ther. 2016;46(2):71-78. PubMed ID: 26721228 doi:10.2519/jospt.2016.6048

18. Hodges P. Transversus abdominis: a different view of the elephant. $\mathrm{Br}$ J Sports Med. 2008;42(12):941-944. PubMed ID: 19096017 doi:10. 1136/bjsm.2008.051037

19. Smith JA, Kulig K. Altered multifidus recruitment during walking in young asymptomatic individuals with a history of low back pain. J Orthop Sports Phys Ther. 2016;46(5):365-374. PubMed ID: 26999410 doi:10.2519/jospt.2016.6230

20. Wilkerson GB, Giles JL, Seibel DK. Prediction of core and lower extremity strains and sprains in collegiate football players: a preliminary study. J Athl Train. 2012;47(3):264-272. PubMed ID: 22892407 doi:10.4085/1062-6050-47.3.17

21. Bagherian S, Ghasempoor K, Rahnama N, Wikstrom EA. The effect of core stability training on functional movement patterns in collegiate athletes. J Sport Rehab. 2019;28(5):444-449. doi:10.1123/jsr. 2017-0107

22. Imai A, Imai T, Iizuka S, Kaneoka K. A trunk stabilization exercise warm-up may reduce ankle injuries in junior soccer players. Int $J$ Sports Med. 2018;39(04):270-274. doi:10.1055/s-0044-100923

23. Whittaker JL, McLean L, Hodder J, Warner MB, Stokes MJ. Association between changes in electromyographic signal amplitude and abdominal muscle thickness in individuals with and without lumbopelvic pain. J Orthop Sports Phys Ther. 2013;43(7):466-477. PubMed ID: 23633621 doi:10.2519/jospt.2013.4440

24. Wirth K, Hartmann H, Mickel C, Szilvas E, Keiner M, Sander A. Core stability in athletes: a critical analysis of current guidelines. Sports Med. 2017;47(3):401-414. PubMed ID: 27475953 doi:10.1007/ s40279-016-0597-7

25. Moseley GL. Evidence for a direct relationship between cognitive and physical change during an education intervention in people with chronic low back pain. Eur J Pain. 2004;8(1):39-45. PubMed ID: 14690673 doi:10.1016/S1090-3801(03)00063-6

26. O'sullivan PB, Phyty GDM, Twomey LT, Allison GT. Evaluation of specific stabilizing exercise in the treatment of chronic low back pain with radiologic diagnosis of spondylolysis or spondylolisthesis. Spine. 1997;22(24):2959-2967. doi:10.1097/00007632-199712150-00020

27. Norris C, Matthews M. The role of an integrated back stability program in patients with chronic low back pain. Complement Ther Clin Pract. 2008;14(4):255-263. PubMed ID: 18940712 doi:10. 1016/j.ctcp.2008.06.001
28. Koumantakis GA, Watson PJ, Oldham JA. Trunk muscle stabilization training plus general exercise versus general exercise only: randomized controlled trial of patients with recurrent low back pain. Phys Ther. 2005;85(3):209-225. PubMed ID: 15733046 doi:10.1093/ptj/ 85.3.209

29. Kavcic N, Grenier S, McGill SM. Determining the stabilizing role of individual torso muscles during rehabilitation exercises. Spine. 2004;29(11):1254-1265. PubMed ID: 15167666 doi:10.1097/ 00007632-200406010-00016

30. Koppenhaver SL, Hebert JJ, Fritz JM, Parent EC, Teyhen DS, Magel JS. Reliability of rehabilitative ultrasound imaging of the transversus abdominis and lumbar multifidus muscles. Arch Phys Med Rehabil. 2009;90(1):87-94. PubMed ID: 19154834 doi:10.1016/j.apmr.2008. 06.022

31. Teyhen DS, Gill NW, Whittaker JL, Henry SM, Hides JA, Hodges P. Rehabilitative ultrasound imaging of the abdominal muscles. J Orthop Sports Phys Ther. 2007;37(8):450-466. PubMed ID: 17877281 doi:10.2519/jospt.2007.2558

32. Wallwork TL, Hides JA, Stanton WR. Intrarater and interrater reliability of assessment of lumbar multifidus muscle thickness using rehabilitative ultrasound imaging. J Orthop Sports Phys Ther. 2007;37(10):608-612. PubMed ID: 17970407 doi:10.2519/jospt.2007.2418

33. Hebert JJ, Koppenhaver SL, Teyhen DS, Walker BF, Fritz JM. The evaluation of lumbar multifidus muscle function via palpation: reliability and validity of a new clinical test. Spine. 2015;15(6): 1196-1202. doi:10.1016/j.spinee.2013.08.056

34. Vleeming A. Second interdisciplinary world congress on low back pain. The Integrated Function of the Lumbar Spine and Sacroiliac Joints (pp. 149-168). San Diego, CA: ECO; 1995.

35. Vleeming A, Pool-Goudzwaard A, Stoeckart R, Wingerden van JP, Snijders CJ. The posterior layer of the thoracolumbar fascia. Its function in load transfer from spine to legs. Spine. 1993;20(7): 753-758. doi:10.1097/00007632-199504000-00001

36. Page P, Frank C, Lardner R. Assessment and Treatment of Muscle Imbalance: The Janda Approach. Champaign, IL: Human Kinetics; 2010.

37. Kibler WB, Press J, Sciascia A. The role of core stability in athletic function. Sports Med. 2006;36(3):189-198. PubMed ID: 16526831 doi:10.2165/00007256-200636030-00001

38. Myers TW. Anatomy Trains:Myofascial Meridians for Manual and Movement Therapists. New York, NY: Elsevier, Churchill Livingstone; 2013.

39. Wilke J, Krause F, Vogt L, Banzer W. What is evidence-based about myofascial chains: a systematic review. Arch Phys Med Rehabil. 2016;97(3):454-461. PubMed ID: 26281953 doi:10.1016/j.apmr. 2015.07.023

40. Kiesel KB, Uhl TL, Underwood FB, Rodd DW, Nitz AJ. Measurement of lumbar multifidus muscle contraction with rehabilitative ultrasound imaging. Man Ther. 2007;12(2):161-166. PubMed ID: 16973400 doi:10.1016/j.math.2006.06.011

41. Ferreira PH, Ferreira ML, Hodges PW. Changes in recruitment of the abdominal muscles in people with low back pain: ultrasound measurement of muscle activity. Spine. 2004;29(22):2560-2566. PubMed ID: 15543074 doi:10.1097/01.brs.0000144410.89182.f9

42. Hides JA, Stanton WR, Mendis MD, Gildea J, Sexton MJ. Effect of motor control training on muscle size and football games missed from injury. Med Sci Sports Exerc. 2012;44(6):1141-1149. PubMed ID: 22157811 doi:10.1249/MSS.0b013e318244a321

43. Hides JA, Stanton WR. Can motor control training lower the risk of injury for professional football players? Med Sci Sports Exerc. 2014;46(4):762-768. PubMed ID: 24056268 doi:10.1249/MSS. 0000000000000169 
44. Brumitt J, Dale RB. Integrating shoulder and core exercises when rehabilitating athletes performing overhead activities. $N$ Am J Sports Phys Ther. 2009;4(3):132-138. PubMed ID: 21509108

45. Sweeney N, O'sullivan C, Kelly G. Multifidus muscle size and percentage thickness changes among patients with unilateral chronic low back pain (CLBP) and healthy controls in prone and standing. Man Ther. 2014;19(5):433-439. PubMed ID: 24909431 doi:10.1016/ j.math.2014.04.009

46. Mangum LC, Sutherlin MA, Saliba SA, Hart JM. Reliability of ultrasound imaging measures of transverse abdominis and lumbar multifidus in various positions. $P M \& R$. 2016;8(4):340-347. doi:10. 1016/j.pmrj.2015.09.015

47. Landis JR, Koch GG. The measurement of observer agreement for categorical data. Biometrics. 1977;33(1):159-174. doi:10.2307/2529310

48. Lederman E. The myth of core stability. J Bodywork Movement Ther. 2010;14(1):84-98. doi:10.1016/j.jbmt.2009.08.001

49. Willardson JM. Core stability training for healthy athletes: a different paradigm for fitness professionals. Strength Cond J. 2007;29(6):42.

50. McGill S. Core training: evidence translating to better performance and injury prevention. Strength Cond J. 2010;32(3):33-46. doi:10. 1519/SSC.0b013e3181df4521

51. Akbari A, Khorashadizadeh S, Abdi G. The effect of motor control exercise versus general exercise on lumbar local stabilizing muscles thickness: randomized controlled trial of patients with chronic low back pain. J Back Musculoskelet Rehabil. 2008;21(2):105-112. doi:10.3233/BMR-2008-21206

52. Mannion A, Caporaso F, Pulkovski N, Sprott H. Spine stabilisation exercises in the treatment of chronic low back pain: a good clinical outcome is not associated with improved abdominal muscle function. Eur Spine J. 2012;21(7):1301-1310. PubMed ID: 22270245. doi:10. 1007/s00586-012-2155-9

53. Mannion AF, Pulkovski N, Gubler D, et al. Muscle thickness changes during abdominal hollowing: an assessment of between-day measurement error in controls and patients with chronic low back pain. Eur Spine J. 2008;17(4):494-501. PubMed ID: 18196294 doi:10. 1007/s00586-008-0589-X

54. Vasseljen O, Fladmark AM, Westad C, Torp HG. Onset in abdominal muscles recorded simultaneously by ultrasound imaging and intramuscular electromyography. J Electromyogr Kinesiol. 2009; 19(2):e23-e31. PubMed ID: 17897842 doi:10.1016/j.jelekin.2007. 07.013

55. Vasseljen O, Unsgaard-Tondel M, Westad C, Mork PJ. Effect of core stability exercises on feed-forward activation of deep abdominal muscles in chronic low back pain: a randomized controlled trial. Spine. 2012;37(13):1101-1108. PubMed ID: 22146280 doi:10.1097/ BRS.0b013e318241377c

56. Koppenhaver SL, Fritz JM, Hebert JJ, et al. Association between changes in abdominal and lumbar multifidus muscle thickness and clinical improvement after spinal manipulation. J Orthop Sports Phys Ther. 2011;41(6):389-399. PubMed ID: 21471653 doi:10.2519/ jospt.2011.3632

57. Moore KL, Dalley AF, Agur AM. Clinically Oriented Anatomy. Philadelphia, PA: Lippincott Williams \& Wilkins; 2013.

58. Juker D, McGill S, Kropf P, Steffen T. Quantitative intramuscular myoelectric activity of lumbar portions of psoas and the abdominal wall during a wide variety of tasks. Med Sci Sports Exerc. 1998; 30(2):301-310. PubMed ID: 9502361 doi:10.1097/00005768199802000-00020

59. McGill S, Juker D, Kropf P. Appropriately placed surface EMG electrodes reflect deep muscle activity (psoas, quadratus lumborum, abdominal wall) in the lumbar spine. J Biomech. 1996;29(11):15031507. PubMed ID: 8894932 doi:10.1016/0021-9290(96)84547-7
60. Hodges PW. Changes in motor planning of feedforward postural responses of the trunk muscles in low back pain. Exp Brain Res. 2001;141(2):261-266. PubMed ID: 11713638 doi:10.1007/s002210 100873

61. Lehman GJ, Vernon H, McGill SM. Effects of a mechanical pain stimulus on erector spinae activity before and after a spinal manipulation in patients with back pain: a preliminary investigation. $J$ Manipulative Physiol Ther. 2001;24(6):402-406. PubMed ID: 11514817 doi:10.1067/mmt.2001.116421

62. Hodges PW, Richardson CA. Altered trunk muscle recruitment in people with low back pain with upper limb movement at different speeds. Arch Phys Med Rehabil. 1999;80(9):1005-1012. PubMed ID: 10489000 doi:10.1016/S0003-9993(99)90052-7

63. Partner SL, Sutherlin MA, Acocello S, Saliba SA, Magrum EM, Hart JM. Changes in muscle thickness after exercise and biofeedback in people with low back pain. J Sport Rehab. 2014;23(4):307-318. doi:10.1123/JSR.2013-0057

64. Urquhart DM, Hodges PW, Allen TJ, Story IH. Abdominal muscle recruitment during a range of voluntary exercises. Man Ther. 2005; 10(2):144-153. PubMed ID: 15922235 doi:10.1016/j.math.2004. 08.011

65. Hides JA, Boughen CL, Stanton WR, Strudwick MW, Wilson SJ. A magnetic resonance imaging investigation of the transversus abdominis muscle during drawing-in of the abdominal wall in elite Australian football league players with and without low back pain. J Orthop Sports Phys Ther. 2010;40(1):4-10. PubMed ID: 20044702 doi:10.2519/jospt.2010.3177

66. Stokes IA, Gardner-Morse MG, Henry SM. Abdominal muscle activation increases lumbar spinal stability: analysis of contributions of different muscle groups. Clin Biomech. 2011;26(8):797-803. doi:10.1016/j.clinbiomech.2011.04.006

67. Grenier SG, McGill SM. Quantification of lumbar stability by using 2 different abdominal activation strategies. Arch Phys Med Rehabil. 2007;88(1):54-62. PubMed ID: 17207676 doi:10.1016/j.apmr.2006. 10.014

68. Bourne MN, Timmins RG, Opar DA, et al. An evidence-based framework for strengthening exercises to prevent hamstring injury. Sports Med. 2018;48(2):251-267. PubMed ID: 29116573 doi:10. 1007/s40279-017-0796-x

69. Raney NH, Teyhen DS, Childs JD. Observed changes in lateral abdominal muscle thickness after spinal manipulation: a case series using rehabilitative ultrasound imaging. J Orthop Sports Phys Ther. 2007;37(8):472-479. PubMed ID: 17877283 doi:10.2519/jospt. 2007.2523

70. Gill NW, Teyhen DS, Lee IE. Improved contraction of the transversus abdominis immediately following spinal manipulation: a case study using real-time ultrasound imaging. Man Ther. 2007;12(3):280-285. PubMed ID: 16971162 doi:10.1016/j.math.2006.06.014

71. Brenner AK, Gill NW, Buscema CJ, Kiesel K. Improved activation of lumbar multifidus following spinal manipulation: a case report applying rehabilitative ultrasound imaging. J Orthop Sports Phys Ther. 2007; 37(10):613-619. PubMed ID: 17970408 doi:10.2519/jospt.2007.2470

72. Pillastrini P, Ferrari S, Rattin S, Cupello A, Villafañe JH, Vanti C. Exercise and tropism of the multifidus muscle in low back pain: a short review. J Phys Ther Sci. 2015;27(3):943-945. PubMed ID: 25931765 doi:10.1589/jpts. 27.943

73. Willemink MJ, van Es HW, Helmhout PH, Diederik AL, Kelder JC, van Heesewijk JP. The effects of dynamic isolated lumbar extensor training on lumbar multifidus functional cross-sectional area and functional status of patients with chronic nonspecific low back pain. Spine. 2012;37(26):E1651-E1658. PubMed ID: 23023592 doi:10.1097/BRS.0b013e318274fb2f 
74. Reese NB. Muscle and Sensory Testing-E-book. St. Louis, MO: Elsevier Health Sciences; 2013.

75. Gross JM, Fetto J, Rosen E. Musculoskeletal Examination. Hoboken, NJ: John Wiley \& Sons; 2015.

76. MacDonald DA, Dawson AP, Hodges PW. Behavior of the lumbar multifidus during lower extremity movements in people with recurrent low back pain during symptom remission. J Orthop Sports Phys Ther. 2011;41(3):155-164. doi:10.2519/jospt.2011.3410

77. Yahia L, Rhalmi S, Newman N, Isler M. Sensory innervation of human thoracolumbar fascia: an immunohistochemical study. Acta Orthop Scand. 1992;63(2):195-197. PubMed ID: 1590057 doi:10. 3109/17453679209154822

78. Stecco C, Porzionato A, Lancerotto L, et al. Histological study of the deep fasciae of the limbs. J Body Mov Ther. 2008;12(3):225-230. doi:10.1016/j.jbmt.2008.04.041

79. Andersson EA, Nilsson J, Ma Z, Thorstensson A. Abdominal and hip flexor muscle activation during various training exercises. Eur J Appl Physiol Occup Physiol. 1997;75(2):115-123. PubMed ID: 9118976 doi:10.1007/s004210050135

80. Andersson EA, Ma Z, Thorstensson A. Relative EMG levels in training exercises for abdominal and hip flexor muscles. Scand $J$ Rehabil Med. 1998;30(3):175-183. PubMed ID: 9782545 doi:10. 1080/003655098444110

81. Axler CT, McGill SM. Low back loads over a variety of abdominal exercises: searching for the safest abdominal challenge. Med Sci Sports Exerc. 1997;29(6):804-811. PubMed ID: 9219209 doi:10. 1097/00005768-199706000-00011
82. Escamilla RF, Babb E, DeWitt R, et al. Electromyographic analysis of traditional and nontraditional abdominal exercises: implications for rehabilitation and training. Phys Ther. 2006;86(5):656-671. PubMed ID: $16649890 \mathrm{doi}: 10.1093 / \mathrm{ptj} / 86.5 .656$

83. Escamilla RF, Lewis C, Bell D, et al. Core muscle activation during swiss ball and traditional abdominal exercises. J Orthop Sports Phys Ther. 2010;40(5):265-276. PubMed ID: 20436242 doi:10.2519/ jospt.2010.3073

84. Dello Iacono A, Padulo J, Ayalon M. Core stability training on lower limb balance strength. J Sports Sci. 2016;34(7):671-678. doi:10. 1080/02640414.2015.1068437

85. Brito J, Figueiredo P, Fernandes L, et al. Isokinetic strength effects of FIFA's "The 11+" injury prevention training programme. Isokinetics Exerc Sci. 2010;18(4):211-215. doi:10.3233/IES-2010-0386

86. Daneshjoo A, Mokhtar AH, Rahnama N, Yusof A. The effects of injury preventive warm-up programs on knee strength ratio in young male professional soccer players. PLoS One. 2012;7(12): e50979. PubMed ID: 23226553 doi:10.1371/journal.pone. 0050979

87. Hodges P, Pengel L, Herbert R, Gandevia S. Measurement of muscle contraction with ultrasound imaging. Muscle Nerve. 2003;27(6):682692. PubMed ID: 12766979 doi:10.1002/mus.10375

88. van der Horst N, Hoef SV, Otterloo PV, Klein M, Brink M, Backx F. Effective but not adhered to: how can we improve adherence to evidence-based hamstring injury prevention in amateur football? [published online ahead of print December 13, 2018]. Clin J Sport Med. 